NATIONALISER L'EFAVIRENZ : LICENCE OBLIGATOIRE, INVENTION COLLECTIVE ET NEO-DEVELOPPEMENTISME AU BRESIL (2001-2012)

Maurice CASSIER, CERMES-CNRS, Marilena CORREA, IMS-UERJ

\title{
Title : NATIONALIZING EFAVIRENZ: COMPULSORY LICENCE, COLLECTIVE INVENTION AND NEO-DEVELOPMENTALISM IN BRAZIL (2001-2012 )
}

Résumé : Cet article étudie la trajectoire juridique, technologique et industrielle d'un médicament antirétroviral, l'Efavirenz, qui est distribué gratuitement aux patients VIH/Sida par le Ministère de la Santé du Brésil depuis le début des années 2000. En mai 2007, un décret du Président de la République suspend l'exclusivité du propriétaire, Merck, sur l'exploitation de la molécule au Brésil, et autorise la production d'une version générique par les laboratoires locaux. Cette licence obligatoire de l'Etat brésilien vise, simultanément, à pérenniser la politique d'accès universel aux traitements pour lutter contre l'épidémie de sida et à renforcer l'industrie pharmaceutique nationale. La nationalisation de l'Efavirenz donne lieu à l'expérimentation d'une production collective sous la forme d'un consortium industriel et inaugure une politique de partenariats entre laboratoires pharmaceutiques publics et privés.

\begin{abstract}
This article examines the legal, technological and industrial trajectory of an antiretroviral drug, Efavirenz, distributed freely to HIV/Aids patients by the Brazilian Ministry of Health since the early 2000s. In May 2007 a presidential decree suspended the exclusive rights of the patent owner, Merck, to exploitation of the molecule in Brazil, and authorized the production of a generic version by local laboratories. This compulsory license by the Brazilian state is also intended to perpetuate the policy of universal access to treatment, with a view to combatting the Aids epidemic and boosting the country's pharmaceutical industry. Nationalization of Efavirenz has given rise to experimentation with collective production in the form of an industrial consortium, and inaugurated a policy of partnerships between public and private pharmaceutical laboratories.
\end{abstract}

Mots clefs : licence obligatoire ; reverse engineering ; invention collective ; médicament générique ; partenariat public/privé

Key words : compulsory licence; reverse engineering; collective invention; generic medicine; public and private partnership 


\section{INTRODUCTION}

En mai 2007, le président de la République du Brésil signait un décret de licence obligatoire pour autoriser l'exploitation de deux brevets qui sont la propriété de Merck sur un médicament très utilisé dans les trithérapies contre le VIH/sida au Brésil, l'Efavirenz ${ }^{1}$. En vertu de cette licence, le gouvernement était autorisé à importer une version générique du médicament, pour un prix de 0,45 dollar par comprimé contre 1,59 dollar pour le produit breveté vendu par Merck, le temps que les laboratoires pharmaceutiques brésiliens développent en leurs murs la technologie de production et soient à même de prendre le relais pour approvisionner le programme Sida du Ministère de la Santé qui distribuait gratuitement la molécule à 75000 patients (soit 40\% de l'ensemble des patients traités) Le gouvernement attendait de la mise en œuvre de cette licence une réduction substantielle du coût d'achat de cette molécule, économie estimée à 236,8 millions de dollars jusqu'à l'expiration des brevets, en 2012 pour le premier brevet de produit, en 2014 pour le second brevet de procédé. Cette première licence obligatoire du Brésil dans le champ du médicament permettait au Président Lula d'affirmer la prééminence de l'intérêt de la santé publique sur les intérêts commerciaux du pays, en dépit de menaces de rétorsions de la Chambre de Commerce des Etats-Unis sur les investissements directs au Brésil : «Entre l'économie et notre santé, nous choisissons notre santé».

En février 2009, le Ministre de la Santé du Brésil inaugurait les premiers lots d'Efavirenz national produits par le laboratoire fédéral Farmanguinhos à Rio de Janeiro. En septembre 2011, lors d'une visite de l'usine du laboratoire fédéral, nous avons pu suivre la ligne de production de l'Efavirenz, depuis l'arrivée des matières premières pharmaceutiques livrées par trois laboratoires privés brésiliens jusqu'à l'empaquetage des capsules du médicament final, en passant par les bidons d'Efavirenz sous forme de poudre. L'Efavirenz est ainsi devenu un médicament générique national certifié par l'Agence du Médicament, l'ANVISA, dès lors qu'il satisfait les essais conduits sur des volontaires sains qui attestent de sa bioéquivalence avec le médicament propriétaire.

\footnotetext{
${ }^{1}$ Une licence obligatoire autorise l'exploitation d'un brevet sans l'autorisation du propriétaire. Celui-ci conserve toutefois la propriété de son brevet et reçoit des royalties au titre de la licence obligatoire. Le décret brésilien fixe un taux de rémunération de 1,5\% du prix payé par le Ministère de la Santé (article 2).
} 
Le modèle brésilien de lutte contre l'épidémie de VIH/sida conjugue étroitement le droit à la santé, qui est inscrit dans la nouvelle constitution du pays de 1988, et la production locale de médicaments génériques assurée par des laboratoires pharmaceutiques publics et privés (Galvao, 2002, Cassier et Correa, 2003, Teixeira et al, 2003). L'année 1996 fut marquée à la fois par une loi présidentielle qui mit en place le principe de l'accès universel aux médicaments contre le VIH/sida (c'est la loi Sarney de novembre 1996) et par le lancement d'un programme de copie des ARVs par le laboratoire pharmaceutique fédéral de Farmanguinhos . Ces copies étaient tout à fait licites dès lors que les premiers ARVs n'étaient pas brevetés au Brésil, en l'absence de brevets de médicament depuis 1945 pour les brevets de produit, et depuis 1971 pour les brevets de procédé. La possibilité de copier disparut avec la nouvelle loi des brevets, adoptée elle aussi en 1996, qui se conformait aux accords APIC $^{2}$ de l'OMC sur la propriété intellectuelle, accords qui imposent des brevets de médicaments de 20 ans à tous les pays membres (Velasquez et C. Correa, 2004). Dans ce nouveau contexte, la licence obligatoire permettait de lever le droit exclusif du breveté et de nationaliser la molécule qui pouvait désormais être copiée par tous les laboratoires installés sur le territoire brésilien. Ce faisant, le gouvernement entendait garantir l'accès universel et gratuit à l'Efavirenz à un nombre croissant de patients et œuvrer simultanément au renforcement des capacités technologiques et industrielles des laboratoires pharmaceutiques nationaux. Il associait, dans la même mesure, la politique de santé contre l'épidémie de sida et la politique industrielle, sous l'autorité du Ministère de la Santé (Cassier et Correa, 2009).

L'histoire de ce médicament singulier, depuis la délivrance de ses brevets en 1999 par l'INPI du Brésil jusqu'à sa production en routine par un consortium de laboratoires nationaux en 2012, nous permet d'étudier à la fois la fabrication du droit de la propriété intellectuelle et le lancement d'une politique néo-développementiste dans le champ de la santé et du médicament. La mise en oeuvre de la licence obligatoire donna lieu à la constitution d'un consortium industriel qui réunit trois laboratoires privés chargés de développer des technologies de synthèse du principe actif qui sera livré à deux laboratoires publics chargés de la formulation du médicament final. L'Efavirenz contribue ici à reconfigurer l'organisation industrielle de l'industrie brésilienne du médicament, en promouvant des principes de coopération et de mutualisation de la technologie et de la production, dans le contexte d'une économie mixte, publique et privée. Le consortium Efavirenz monté en 2007 inaugure une

\footnotetext{
${ }^{2}$ Aspects des Droits de Propriété Intellectuelle qui touchent au Commerce.
} 
politique de partenariats de développement technologique ${ }^{3}$ qui visent à la fois à fournir le Brésil en médicaments dits «stratégiques » (loi 978 du 28 mai 2008) et à élargir les capacités technologiques et industrielles des laboratoires locaux. Des lois adoptées en 2008 et 2010 permettent à l'Etat de s'émanciper des règles des marchés publics standards pour conclure des «contrats de service » et des «partenariats de développement technologique » avec des laboratoires localisés sur le territoire brésilien pour favoriser la production nationale, y compris si les prix des produits brésiliens sont immédiatement supérieurs de $25 \%$ aux prix des produits étrangers. Ce surplus de prix, autorisé pour une période de 5 ans, est gagé sur des obligations de création de richesses économiques en termes d'emplois et de recettes fiscales ainsi que sur des actions de développement et d'innovation technologiques ${ }^{4}$. On peut ici parler de politique néo-développementiste qui vise à construire « un complexe industriel de santé $»^{5}$ et à réduire la dépendance du Brésil qui importe d'Inde et de Chine la majeure partie des principes actifs qu'il utilise (Cassier et Correa, 2003, Orsi et al, 2003, Gueniff, 2012). La licence obligatoire de l'Efavirenz a ainsi été l'événement déclencheur et le banc d'essai de ces dispositions et de cette nouvelle politique.

Nous analyserons dans ce papier les épreuves juridiques, technologiques et industrielles du processus de nationalisation de l'Efavirenz, depuis les premiers travaux de reverse engineering conduits au laboratoire fédéral en 2001 jusqu'à la production désormais en routine du générique certifié. La durée du processus de nationalisation de ce médicament, soit plus de 10 années, aussi bien sur le versant juridique que sur le versant technologique, est un fait essentiel à considérer. Pour étudier ce processus, nous disposons de trois types de sources : des entretiens avec les chimistes en charge de la duplication et de la production du médicament, des visites sur les sites industriels, ainsi que des documents juridiques. En premier lieu, nous avons collecté à partir de décembre 2002 des entretiens auprès des laboratoires qui étaient déjà engagés dans le reverse engineering de la molécule. Nous avons ensuite réalisé des enquêtes en 2009, 2010 et 2011 auprès des responsables des firmes qui étaient membres du consortium industriel chargé de produire le médicament générique. Nous avons enfin réuni des sources juridiques et réglementaires, qui sont particulièrement nombreuses pour ce médicament, notamment les archives du procès entre le propriétaire du brevet et le laboratoire pharmaceutique fédéral entre 2004 et 2006, le décret de licence

\footnotetext{
${ }^{3}$ Product Development Partnership, PDP.

${ }^{4}$ article 6 de la loi 12349 de décembre 2010.

${ }^{5}$ Loi 742 du Ministère de la Santé de novembre 2007, Lois 374 et 375 de février 2008, décret présidentiel du 12 mai 2008, puis liste de produits stratégiques qui justifient la mise en place du complexe industriel de santé publiée le 16 mai 2008.
} 
obligatoire de mai 2007, les publications au journal officiel de l'appel d'offres constitutif du consortium industriel, les documents contractuels qui gouvernent les obligations et les échanges des partenaires, les contrats de service du Ministère de la Santé qui passent commande des principes actifs pharmaceutiques auprès des laboratoires nationaux privés. Des entretiens approfondis et renouvelés avec les chimistes ont permis d'inventorier les échanges de savoirs, de technologies, de personnel, de matières premières, de produits pharmaceutiques entre les différents partenaires, autrement dit de restituer l'économie concrète de ce collectif industriel.

\section{1) SANTE PUBLIQUE, REVERSE ENGINEERING ET EPREUVES DE FORCES JURIDIQUES (2001-2007)}

Les laboratoires brésiliens, tant publics que privés, s'engagèrent très tôt dans les travaux de reverse engineering de l'Efavirenz, alors que les brevets couvrant la molécule avaient été délivrés par l'INPI du Brésil en 1999, en vertu de la nouvelle loi sur les brevets adoptée par le Parlement en 1996. Tandis que les premiers ARVs ${ }^{6}$ dupliqués par les laboratoires de génériques brésiliens étaient libres de droits de brevet, l'Efavirenz était la propriété exclusive de Merck sur le territoire brésilien. Merck était d'autant plus attachée à son monopole que la firme avait concédé à Bristol Myers Squibb les droits pour la commercialisation de 1'Efavirenz pour les Etats-Unis, le Canada et six pays européens et que les achats du Ministère de la santé du Brésil représentaient une source de revenus non négligeable pour la compagnie. Pour sa part, Le Ministère de la Santé du Brésil encouragea dés l'année 2000 le reverse engineering de la molécule par le laboratoire fédéral de Rio de Janeiro, Farmanguinhos, pour menacer Merck d'une licence obligatoire et faire pression sur les prix : «S'ils consentaient une diminution du prix, nous ne produirions pas sur place, mais s'ils refusaient, nous y étions prêts » (E. Pinheiro, Directrice de Farmanguinhos, mars 2002). Le Ministère de la santé demanda aux laboratoires privés de chimie pharmaceutique de développer la synthèse du principe actif qu'ils pourraient vendre au laboratoire fédéral qui produirait quant à lui la formulation du médicament final : «Quand le gouvernement a manifesté l'intérêt de casser le brevet du Nelfinavir et de l'Efavirenz, nous sommes entrés aussi. Nous avons même soumis un échantillon, nous avons développé le procédé ici »

\footnotetext{
${ }^{6}$ Antirétroviraux
} 
(directeur de la $R \& D^{7}$ de Nortec, mai 2004). Fort des technologies engrangées par les laboratoires brésiliens, en cas d'échec de la négociation commerciale, le Ministère de la Santé pouvait brandir la menace d'une licence obligatoire : « une fois que les fabricants nationaux ont développé le reverse engineering, ils vont parler aux multinationales en toute sécurité «si vous ne nous fournissez pas, j'ai à qui faire recours. On a la connaissance, le know how. » (Directeur de la R\&D, Cristalia, avril 2004).

Les progrès du reverse engineering de l'Efavirenz dans les laboratoires brésiliens ne manquèrent pas de susciter l'opposition de Merck. En 2001, la firme adresse une lettre de mise en garde au laboratoire fédéral pour lui défendre de se procurer les matières premières de l'Efavirenz en Inde. Farmanguinhos répond que la loi brésilienne sur les brevets l'autorise à effectuer des recherches sur le médicament importé dès lors qu'il ne fait pas l'objet d'un commerce, mais qu'il est objet de recherche. En 2004, la confrontation s'aiguise quand l'Agence de Sécurité Sanitaire du Brésil enregistre 3 formulations de médicament « similaire » développés par des laboratoires nationaux et quand le laboratoire fédéral publie un appel d'offres pour se procurer $200 \mathrm{Kg}$ de matières premières d'Efavirenz.. Deux rationalités s'affrontent. Le laboratoire fédéral brésilien défend la possibilité de commencer des travaux de R\&D sur un médicament breveté pour obtenir l'enregistrement du médicament générique auprès de l'Agence de Sécurité Sanitaire, sans attendre l'expiration du brevet (c'est ce qu'on appelle «l'exemption Bolar» du nom d'une jurisprudence des Etats-Unis de 1984 qui est inscrite dans la loi brésilienne). Farmanguinhos explique que l'achat des $200 \mathrm{~kg}$ de matières premières auprès d'un génériqueur indien est destiné à la recherche et permettra de produire des lots à l'échelle pilote. Il s'agit de «maîtriser un nouveau know how pour le transformer en une nouvelle ligne de production qui pourra être effective à l'expiration du brevet en $2012 »^{8}$. Farmanguinhos justifie également le lancement de son appel d'offres par l'inertie de Merck qui tarde à négocier une licence volontaire. Merck oppose au laboratoire fédéral son monopole sur la production et le commerce de l'Efavirenz. La firme propriétaire déclare que l'appel d'offres sur une molécule brevetée est illicite dès lors qu'elle est la seule à pouvoir la commercialiser. L'appel d'offres du laboratoire brésilien est assimilé à «une licence obligatoire du produit breveté, illégale et inconcevable $»^{9}$. L'appel d'offres est suspendu quelques mois et les négociations pour une licence volontaire reprennent : une équipe de Merck visite les installations du laboratoire fédéral. Finalement, Merck propose un

\footnotetext{
${ }^{7}$ Recherche et Développement

${ }^{8}$ Lettre de la Directrice de Farmanguinhos , Nubia Boechat du 15 octobre 2004.

${ }^{9}$ Acte de contestation de l'appel d'offres de Merck du 18 février 2005.
} 
prix jugé trop élevé par Farmanguinhos et annonce qu'elle ne pourra fournir la quantité demandée : «étant donné la hausse du nombre de patients et l'augmentation conséquente de la demande mondiale de Stocrin ${ }^{10} \gg$ (lettre du 17 février 2005). Le laboratoire Fédéral reprend la procédure d'appel d'offres pour s'approvisionner en Inde et Merck porte l'affaire en justice.

L'issue de ce procès est décisive dès lors que les juges fédéraux, après avoir suivi dans un premier temps l'argument de Merck, se rangent derrière celui du laboratoire fédéral en août 2005 puis en appel en décembre 2006. Les juges font valoir l'intérêt de la santé publique et l'urgence qu'il a à acquérir la technologie du médicament pour ne pas retarder l'arrivée des génériques auprès des malades : «le retard du développement des recherches susmentionnées provoquera le retard de l'acquisition de la technologie et de la fabrication des médicaments génériques ...le retard ou l'interdiction de la production portera préjudice à la santé publique à cause du manque des médicaments génériques sur le marché $»^{11}$. Cette décision inscrit l'exemption Bolar dans la jurisprudence brésilienne et elle ouvre la voie pour une licence obligatoire. En décembre 2006, le Directeur Général de FarManguihnos encourage le gouvernement à décider d'une licence obligatoire : il peut produire l'Efavirenz $50 \%$ moins cher que le prix proposé par Merck. Entre temps, le procès engagé par Merck en février 2005 avait arrêté les recherches sur l'Efavirenz dans les laboratoires brésiliens pendant deux années $^{12}$.

Le décret présidentiel de licence obligatoire de l'Efavirenz de mai 2007 a été préparé par un intense travail de R\&D au sein de plusieurs laboratoires brésiliens, publics et privés. Dès 2004, le Ministère de la Santé envisage un scénario de licence obligatoire qui prévoit à la fois l'importation du médicament générique pour ne pas rompre l'approvisionnement du médicament pour le Programme Sida, et l'implantation d'une production nationale. Un tableau confidentiel dresse une liste des laboratoires indiens susceptibles d'approvisionner les importations ainsi qu'une liste de laboratoires nationaux capables de fournir le principe actif (Cristalia, Nortec et Labogen) et le médicament final (3 laboratoires publics Farmanguinhos , LAFEPE, FURPE, et un laboratoire privé, Cristalia). La bataille se déroule également sur la scène politique avec la mobilisation des ONGs internationales et des associations de patients VIH/Sida qui font campagne pour l'accès aux traitements (Possas, 2008, Flynn, 2007, Chaves $\&$ al., 2008, Rosenberg, 2010) . En avril 2003, le responsable de la campagne pour l'accès aux

\footnotetext{
${ }^{10}$ Stocrin est la marque de l'Efavirenz commercialisé par Merck.

${ }^{11}$ Décision du 6 octobre 2005, Tribunal Régional Fédéral de la $2^{\text {ème }}$ Région.

${ }^{12}$ Entretien avec le directeur adjoint de Farmanguinhos, J. Costa, en mai 2009.
} 
traitements de MSF au Brésil, Michel Lostrowska, organisa un forum international à Rio de Janeiro pour préparer une décision de licence obligatoire. Le forum réunissait des ONGs internationales, comme Oxfam ou James Love du CPtech des Etats-Unis, l'Institut Farmanguinhos qui produisait les ARVs pour le programme sida, la Fondation Oswaldo Cruz, l'Institut National de la Propriété industrielle, le syndicat de l'Industrie chimique du Brésil, des économistes de l'Université Fédérale de Rio, des responsables du programme sida du Brésil, des juristes spécialisés en propriété intellectuelle. Ce forum était emblématique de la nouvelle gouvernance pharmaceutique brésilienne (Biehl, 2009) qui implique les laboratoires de génériques, les scientifiques, les ONGs, des juristes et l'Etat. En 2006, MSF Brésil et l'association de patients ABIA commandèrent une expertise conduite par deux chimistes universitaires pour évaluer les capacités industrielles des laboratoires brésiliens ${ }^{13}$. Le résultat favorable de l'expertise permit de lever les doutes qui subsistaient sur les capacités technologiques et industrielles des laboratoires locaux à produire une molécule aussi sophistiquée que l'Efavirenz.

Dès le printemps 2007, le laboratoire fédéral de Farmanguinhos prépare la mise en place de la structure industrielle pour produire le médicament générique. Le 3 avril 2007, avant même le décret de licence obligatoire du 7 mai, le laboratoire fédéral ouvre un appel d'offres pour la pré-qualification des laboratoires privés qui seront chargés de produire le principe actif de l'Efavirenz. Un directeur de Farmanguinhos visite les laboratoires brésiliens pour évaluer leurs capacités de production et rédige un rapport technique qui servira de base à la formation du consortium : «il a rédigé un rapport qui retenait 6 entreprises brésiliennes à même de satisfaire les exigences de la production » (entretien avec la directrice adjointe de $\mathrm{FM}^{14}$, mai 2010). Entre temps, le 24 avril 2007, le Ministère de la Santé publie un décret « d'intérêt public » en vue de prendre une licence obligatoire pour l'Efavirenz. Le décret de licence obligatoire est pris le 4 mai 2007 pour une durée de 5 ans qui peut être renouvelée ${ }^{15}$. Dans la mesure où la licence obligatoire est par définition non exclusive, elle peut bénéficier à tous les laboratoires intéressés implantés sur le territoire national. Au Brésil, elle sera exploitée par un collectif de laboratoires publics et privés unis par un contrat de consortium. Dans la mesure où elle est dédiée à «un usage public et non commercial dans le cadre du programme Sida du Brésil» (article 1 du décret de licence obligatoire), elle est placée sous

\footnotetext{
${ }^{13}$ Antunes O, Fortunak JM, 2006, «Arvs Production in Brazil : an Evaluation », report for the Brazilian Interdisciplinary Aids Association (ABIA) and MSF Brazil, 8 pages.

${ }^{14} \mathrm{FM}$ ou Farmanguinhos, le laboratoire pharmaceutique fédéral.

${ }^{15}$ A l'automne 2011, le laboratoire fédéral consultait les firmes membres du consortium pour le renouvellement de la licence obligatoire prévu en 2012.
} 
l'autorité du Ministère de la Santé. De fait, c'est le laboratoire fédéral de Farmanguinhos qui organise un jury pour auditer les 9 entreprises nationales candidates et sélectionner les 3 firmes qui seront membres du consortium Efavirenz ${ }^{16}$. Le jury est composé de chimistes du laboratoire fédéral, du directeur du laboratoire officiel de l'Etat de Pernambouc, le LAPEPE, d'un consultant extérieur, d'un juriste, d'un membre du syndicat de l'industrie pharmaceutique, ABIFINA. Chacune des 9 firmes est auditionnée sur «sa technologie, le temps qu'elle estime nécessaire pour le développement de la production, sa capacité de production, la prévision de ses coûts, sa capacité en R\&D, etc » (J Costa, mai 2009). Le 24 juillet 2007, le résultat de 1'appel d'offres est publié au Journal Officiel. L'accord de consortium qui lie les 3 laboratoires privés à la Fondation Oswaldo Cruz est signé en décembre 2007, puis renouvelé en $2009^{17}$.

\section{2) ETAT FEDERAL, CONSORTIUM INDUSTRIEL ET INVENTION COLLECTIVE (2007-2012)}

La formation du consortium industriel procède de la licence obligatoire. Il en est l'outil de production. Quelles sont les justifications et la rationalité de l'organisation collective mise en place pour produire ce médicament d'intérêt public?

La stratégie de mutualisation du développement de la technologie et de la production du médicament placé sous licence obligatoire obéit tout d'abord à une rationalité de court terme, celle de l'approvisionnement du programme sida du Brésil avec un médicament générique certifié par l'agence du médicament. Le collectif industriel créé permet d'organiser une complémentarité et une division verticale du travail entre les laboratoires privés, qui produiront le principe actif, et les laboratoires officiels, au départ Farmanguinhos à Rio et le LAFEPE à Recife, qui s'occuperont de la formulation. Dans la mesure où 3 laboratoires privés sont réunis pour produire les principes actifs, chacun à son tour selon un chronogramme, et où deux laboratoires publics sont prévus pour se partager la production du médicament final et sa livraison au programme sida, le consortium répond à un objectif de sécurité des approvisionnements, pour pallier d'éventuelles difficultés ou défaillance de tel ou tel participant. Il s'agit aussi, dans l'esprit des concepteurs du consortium, de stimuler l'amélioration de la technologie au sein des différents laboratoires en escomptant une incitation par un prix fixé : «l'idée est de ne pas dépendre d'un seul fournisseur, mais d'avoir

\footnotetext{
${ }^{16}$ Entretien avec J Costa, mai 2009.

${ }^{17}$ Contrat de consortium n ${ }^{\circ} 32 / 2009$, Ministère de la Santé.
} 
un seul prix ; ce qui pousse à optimiser les procédés » (J Costa,, mai 2009). Le consortium instaure une complémentarité entre les partenaires publics et privés et il assure la sécurité d'un approvisionnement jugé stratégique pour le gouvernement (une défaillance des laboratoires brésiliens ne manquerait pas de ruiner l'utilisation future des licences obligatoires pour des médicaments au Brésil),. Il permet aussi de mobiliser les capacités de R\&D des différents laboratoires qui sont des laboratoires de taille moyenne (le plus important, Cristalia, compte 2200 salariés). Et tous les laboratoires, publics comme privés, ont accès à l'expertise technologique de la Centrale Analytique du laboratoire fédéral Farmanguinhos qui a une mission de distribution des savoirs. Le consortium permet, pour le développement de certaines technologies, de mutualiser les investissements de R\&D dont une partie est prise en charge par l'Etat.

La création du consortium Efavirenz obéit simultanément à une rationalité de moyen et long terme. La duplication d'un médicament aussi sophistiqué que l'Efavirenz suppose un processus d'apprentissage technologique chez les différents participants. Tous les membres du consortium sont conduits à améliorer leurs technologies pour respecter les standards de production définis par le laboratoire fédéral et pour augmenter leur marge sur le prix fixé par le contrat. La participation au consortium permet de se maintenir à la frontière du développement technologique. En répartissant le volume de production de l'Efavirenz entre 3 laboratoires, le gouvernement exprime aussi clairement l'intérêt qu'il a à renforcer un groupe ou cluster de laboratoires nationaux capables de produire des matières premières pharmaceutiques pour lesquelles l'industrie brésilienne est fortement dépendante des fournisseurs indiens ou chinois : «Au Brésil, nous avons un petit nombre de firmes dans ce secteur; beaucoup trop restreint: Nortec, Cristalia, Globe, Cyg Biotech, .. » (J. Costa, directeur de l'innovation de la Fondation Oswaldo Cruz, octobre 2011). Le consortium participe à la consolidation de ce noyau industriel. Les firmes sélectionnées vont bénéficier d'avantages économiques - augmenter leur production et vendre des matières premières sophistiquées au laboratoire fédéral- et d'avantages technologiques - ce sont les effets d'apprentissage liés à la duplication d'une molécule complexe.

L'accord de consortium réunit les 3 laboratoires privés en une organisation collective, le consortium CNG, qui contracte avec la Fondation Oswaldo Cruz pour l'approvisionner en principe actif selon les spécifications techniques fixées par le laboratoire fédéral. Ce collectif est hiérarchisé : un laboratoire, Cristalia, est désigné comme « le laboratoire leader ». Il est vrai qu'il est d'une taille supérieure aux autres, qu'il possède des capacités de R\&D également supérieures et qu'il peut aussi bien produire le principe actif que le médicament 
final pour le cas échéant suppléer le laboratoire fédéral. La première obligation des entreprises membres du consortium est de posséder une usine sur le territoire national dans laquelle sera effectuée la production du principe actif. Cette obligation de production domestique répond à la fois au droit de la licence obligatoire, qui n'est valide que sur le territoire brésilien, et à la politique du gouvernement de développer la production nationale de principes actifs pharmaceutiques. Toutes les opérations de production, de circulation et de stockage du principe actif sont codifiées et programmées par le contrat - depuis l'arrivée des matières premières achetées par les firmes, les procédés industriels de transformation qu'elles utilisent, les bonnes pratiques de fabrication qu'elles doivent satisfaire, jusqu'aux conditions de stockage, de manipulation, de transport des produits finis pour garantir leur bonne qualité. Le contrat structure et encadre la gestion de la production des firmes contractantes par un réglage précis de la circulation des produits et des informations techniques. Ainsi les producteurs du principe actif doivent-ils communiquer toute leur documentation technique au laboratoire fédéral. Tous les incidents de production doivent être immédiatement communiqués à tous les membres du consortium et ceux-ci sont tenus de répondre aux demandes d'information du laboratoire fédéral : «les entreprises membres du consortium doivent fournir toutes les informations relatives à ces difficultés demandées par la partie contractante - la Fondation Oswaldo Cruz » (article 7-10). Le Laboratoire fédéral doit pour sa part tenir un registre des incidents de production et le communiquer aux firmes (article 6). Le Laboratoire fédéral concentre toutes les informations techniques, surveille et dirige la production, par les spécifications techniques qu'il fixe et auxquelles les firmes doivent se conformer et par la surveillance directe qu'il exerce par ses services techniques (article 10). En cas de difficultés, si les firmes participantes proposent des solutions de correction, le laboratoire fédéral doit donner son accord pour que la production puisse reprendre. Cette circulation des informations techniques, pour la production en routine et lors des difficultés de production, renforce l'intégration du collectif industriel, sous l'autorité du laboratoire public. A cette circulation verticale se superposent des circulations horizontales entre les firmes privées, qui doivent échanger les informations sur les incidents de production, et qui ont également le pouvoir de s'arranger entre elles pour se répartir la production au cas où l'une d'entre elles viendrait à se retirer du consortium (art 7.11).

Au-delà des obligations contractuelles, quelle est l'économie réelle des échanges au sein du consortium au regard des récits recueillis auprès du laboratoire fédéral et des laboratoires privés? Quel est le degré de mutualisation des savoirs dans un collectif qui réunit à la fois des firmes concurrentes (les 3 producteurs de principe actif) et des firmes 
complémentaires (les laboratoires producteurs de principe actif, d'un côté, et les laboratoires producteurs du médicament final, d'un autre côté) ? On peut distinguer plusieurs situations qui font la part plus ou moins belle à la coopération ou aux travaux séparés.

La première situation concerne le développement des technologies de synthèse pour être à même de produire le principe actif au Brésil. Ces technologies sont développées de manière séparée par les 3 firmes privées dans le contexte de la présélection des membres du consortium, au printemps 2007. Seul le laboratoire fédéral a une vue complète des différents procédés développés par les firmes concurrentes, connaissance qu'il se garde bien de mettre en circulation pour respecter l'espace réservé de chaque industriel : "Chaque compagnie avait la liberté de choisir les variantes du procédé, dès lors que la qualité finale répondait aux spécifications du laboratoire fédéral ; les chimistes du laboratoire fédéral visitaient chaque compagnie, Cristalia, Globe et Nortec; ils avaient une connaissance des trois procédés développés ; pour ma part, je n'avais pas une vue complète des procédés des autres firmes » (PDG de Nortec, mai 2010). Lors des réunions du consortium, le laboratoire fédéral mutualisait certaines données tandis qu'il respectait des espaces de confidentialité pour des données plus sensibles: "nous avions des réunions du consortium; mais les échanges d'information se faisaient de manière séparée avec chaque firme; nous avions aussi des échanges communs : par exemple, les 3 firmes utilisent le même intermédiaire, le trisphogène. Je sais que Cristalia utilise 2 des 3 intermédiaires que j'utilise; le $3^{\text {ème }}$ est différent; pour Globe, je ne connais pas le sel qu'ils emploient. Nous avions défini des variables essentielles du procédé qui sont les mêmes » (Nortec, mai 2010). Le consortium organise ainsi des espaces communs et des espaces privés pour l'échange des technologies ${ }^{18}$.

La seconde situation de coopération concerne les technologies d'analyse de la molécule. Ces technologies ont d'emblée un statut de «bien commun » dès lors qu'elles sont développées par le laboratoire fédéral qui les distribue à tous les laboratoires publics et privés. Il faut savoir que Farmanguinhos dispose d'un département d'analyse bien équipé qui joue le rôle de plateforme technologique pour l'industrie pharmaceutique brésilienne. Le développement de cette technologie ne fut pas aisé dès lors que le brevet de Merck s'avéra singulièrement lacunaire : «la méthode d'analyse décrite dans le brevet de Merck ne correspond pas au produit » (réunion en mai 2009 au laboratoire fédéral avec l'équipe de R\&D). Pour pallier les insuffisances du brevet, les chimistes réutilisèrent les travaux qu'ils

\footnotetext{
${ }^{18}$ Cette gestion des espaces privés et collectifs au sein du consortium est similaire à celle que nous avions décrite dans les consortiums de recherche en biotechnologie, Cassier 1998: « Le partage des connaissances dans les réseaux scientifiques », Revue Française de Sociologie.
} 
avaient conduit en 2002 pour élaborer le standard de la molécule pour le compte de l'Agence de Sécurité Sanitaire, l'ANVISA. Une fois développée, Farmanguinhos transféra la méthode d'analyse et les spécifications du produit aux 3 firmes du consortium. Deux laboratoires privés envoyèrent du personnel à Farmanguinhos pour $\mathrm{y}$ apprendre cette méthode et Farmanguinhos délégua également ses chimistes dans les entreprises. Ce partage de la technologie renvoie à la mission du laboratoire fédéral. C'est aussi un moyen de faire converger les 3 laboratoires privés vers les mêmes spécifications du produit. Le laboratoire fédéral procéda au test des échantillons de produits des trois industriels. Les résultats des tests étaient remis aux laboratoires dans des réunions du consortium, ou de manière séparée avec chacun d'entre eux pour des informations plus confidentielles. Au-delà des résultats des tests, Farmanguinhos a parfois suggéré des améliorations possibles des procédés de synthèse utilisés par les producteurs privés. Le consortium offre ici un cadre d'apprentissage collectif et de perfectionnement de la technologie.

Le lancement puis la montée à l'échelle de la production du médicament sous licence obligatoire ont ensuite favorisé des processus de mutualisation des savoirs entre les firmes, particulièrement lors des phases de crise de la production.

Conformément à l'esprit et à la lettre de l'accord de consortium, Farmanguinhos assura un rôle de suivi de la production auprès des laboratoires privés et centralisa toutes les informations techniques. Une fois le consortium installé, FM créa une commission de suivi de la production du principe actif qui comprenait deux bras : un bras logistique pour suivre et lever tous les problèmes d'approvisionnement que les laboratoires privés pourraient rencontrer; et un bras technique pour résoudre les difficultés qui pourraient survenir pour la production. Cette commission technique comprend 4 personnes issues des départements de la production, du développement et de la qualité de Farmanguinhos ${ }^{19}$. Cette commission a été très active dans la phase de démarrage de la production et lors des crises de production, puis ses réunions se sont espacées. Les laboratoires industriels nous ont décrit une surveillance étroite de leur production par le laboratoire fédéral dès lors qu'un « contrat de service $»^{20}$ a été conclu entre les deux parties pour approvisionner Farmanguinhos : «nous devons acquérir la

\footnotetext{
${ }^{19}$ Entretien avec J. Mendonça, FM, septembre 2011.

${ }^{20}$ Les contrats de service ont été mis en place par une loi en 2008 qui autorise le Ministère de la Santé à acquérir des matières premières pharmaceutiques sans utiliser la procédure standard des marchés publics qui obéit à la recherche du prix le plus bas, le cas échéant au détriment de la qualité des produits. Les contrats de service sont conclus pour 5 années avec un laboratoire établi sur le territoire national pour acquérir un principe actif dont on s'efforce de garantir la qualité.
} 
matière première, l'intermédiaire, et ensuite Farmanguinhos passe contrat pour la production et contrôle effectivement la production. Ils viennent contrôler l'analyse de la matière première, ils viennent pour contrôler la production et ils viennent pour vérifier l'analyse du principe actif. Ils suivent réellement tout le processus productif » (directeur de Nortec, mai 2010).

Les crises de production et leur résolution ont accéléré un processus de rapprochement et d'apprentissage collectif entre les firmes du consortium. Les premières difficultés sont apparues en 2008 lors des essais de bioéquivalence pour l'enregistrement de l'Efavirenz du laboratoire fédéral comme médicament générique. Le premier essai sur 60 volontaires sains n'a pas donné les résultats escomptés et les chercheurs de Farmanguinhos ont dû remonter en amont pour modifier la formulation ainsi que la méthode d'analyse de la molécule (dans un premier temps, les chimistes brésiliens avaient utilisé la méthode indiquée par Merck dans son brevet et celle-ci s'est avérée insuffisamment fiable pour évaluer les produits ${ }^{21}$. Le second essai fut satisfaisant et l'Efavirenz brésilien fut certifié comme médicament générique par l'agence brésilienne du médicament, l'ANVISA. Au cours de l'année 2010, Farmanguinhos a rencontré de sérieuses difficultés de production au point qu'il a été nécessaire d'arrêter la fabrication pendant plusieurs mois. Entre temps, le laboratoire avait perdu des lots de produits. Les problèmes se sont manifestés principalement avec les livraisons de principe actif d'un des 3 fournisseurs (le consortium prévoyait des livraisons échelonnées dans le temps : avec le premier fournisseur, FM n'a rencontré aucune difficulté ; avec le second, quelques lots posent des problèmes ; avec le $3^{\text {ème }}$, la production doit s'arrêter). Les chimistes font plusieurs hypothèses : s'agit-il de molécules polymorphes ou est-ce dû aux conditions de stockage et de transport de la matière première ? La commission technique part visiter les usines et les industriels vinrent à Farmanguinhos pour identifier le problème. Le laboratoire fédéral presse les industriels d'harmoniser leurs routes de synthèse, qui se révèlent très proches les unes des autres. Deux des trois laboratoires décident de s'approvisionner chez le même fournisseur indien pour l'achat d'un intermédiaire clé de la synthèse. Nortec propose son entremise avec un génériqueur indien qui détient une formulation de l'Efavirenz. Il fut question un temps d'échanger avec les chimistes indiens, le cas échéant de les inviter à Farmanguihnos, pour résoudre le problème (entrevue avec la directrice adjointe, mai 2010). Finalement, Farmanguinhos émit l'hypothèse que c'était la taille des cristaux qui était en cause : «nous avons appris, chaque firme a appris que le principe actif devait être «micronisé », la taille du

\footnotetext{
${ }^{21}$ Entretien avec le responsable des essais de bioéquivalence pour l'Efavirenz, A Daher, mai 2010.
} 
cristal devait être réduite. Mais au départ, aucun d'entre nous le savait !» (Cristalia). Le laboratoire fédéral modifia les spécifications et les laboratoires privés ajustèrent leur procédé pour s'y conformer. Au cours de cette crise, les laboratoires privés échangèrent directement entre eux sur la question de la taille des cristaux : « à cette occasion, nous discutions avec les autres firmes qui produisaient le principe actif de l'Efavirenz : rencontrez-vous des difficultés quand vous réduisez la taille des cristaux ? »(Cristalia). Les laboratoires privés furent donc conduits à partager des informations techniques pour surmonter des difficultés de production. Chacun avait appris dans le cours de la production, par un processus de « learning by doing » (Arrow, 1962).

La mutualisation des savoirs s'accrut encore pendant les quelques mois où le laboratoire fédéral arrêta sa production et où il fut remplacé par l'entreprise privée Cristalia pour produire le médicament final. Cette substitution des laboratoires au sein du consortium entraîna un changement de la circulation des produits et des informations techniques : c'est désormais Cristalia qui reçut tous les principes actifs en provenance des autres laboratoires ainsi que la documentation technique afférente. Cette rotation des rôles au sein du collectif industriel s'accompagna d'une mutualisation «de fait » des savoirs techniques. En utilisant les principes actifs fournis par tous les producteurs, Cristalia put démontrer que les difficultés de production rencontrés par le laboratoire fédéral ne venaient pas des caractéristiques des différentes sources de principe actif, mais du procédé de production employé pour fabriquer le médicament final: « les autres laboratoires nous adressèrent leurs principes actifs ; Cristalia ne travaillait pas seulement avec le principe actif qu'elle fabriquait; nous travaillions également avec ceux des autres; et tous marchaient ; tous marchaient! »(Cristalia, 2011).

Dans quelle mesure peut-on parler d'apprentissage collectif sinon «d'invention collective $»^{22}$ au sein du consortium Efavirenz? Tandis que le développement des technologies de synthèse fut réalisé de manière séparée par les laboratoires brésiliens dans le contexte de la sélection des firmes qui concourraient à l'appel d'offres du consortium Efavirenz, la mise en place de l'accord de consortium puis le lancement de la production occasionnèrent des échanges directs entre les firmes privées et entre ces dernières et le laboratoire fédéral. La résolution des difficultés de production nécessita des ajustements

\footnotetext{
${ }^{22}$ La notion d'invention collective fut proposée par R. Allen en 1983 pour caractériser les améliorations des technologies sidérurgiques au sein d'un district industriel anglais au $19^{\text {ème }}$ siècle grâce à un processus de divulgation et de circulation des informations entre les firmes locales. Les échanges entre les firmes brésiliennes pour apprendre et mettre au point la technologie de production de l'Efavirenz, dans un contexte d'urgence nationale, se rapprochent de ce dispositif.
} 
mutuels, l'adoption de nouvelles spécifications du produit, ce qui accéléra le partage des informations techniques, y compris entre firmes privées rivales. La rotation des rôles au sein du consortium, à savoir le remplacement du laboratoire fédéral par le laboratoire Cristalia pour produire le médicament final pendant quelques mois au cours de l'année 2010, renforça encore la mutualisation des informations. Tous ces ajustements pour produire un médicament certifié par l'Agence du médicament furent désormais partagés au sein d'un collectif de firmes brésiliennes dont les capacités technologiques avaient été fortifiées.

Si l'apprentissage collectif est avéré, peut-on parler «d'invention » dès lors qu'il s'agit de reproduire une molécule connue ? En premier lieu, qu'il s'agisse des technologies de synthèse, des méthodes d'analyse ou de formulation, elles ne sont aucunement le résultat d'un simple travail de duplication des informations contenues dans les brevets de Merck qui se sont révélés très lacunaires sur ces différents points. La copie relève d'un travail de « dépaquetage » de la technologie (C. Furtado, 1981) qui consiste à réinventer le savoir manquant dans le brevet (Cassier, Correa, 2003). En second lieu, les voies de synthèse actuellement mises en œuvre par les laboratoires brésiliens ne pourraient pas être celles qui ont été enregistrées par Merck lors du dépôt de son brevet dans les années 1990 tant la technologie a changé depuis. Les chimistes des firmes brésiliennes ont pu accéder à ces perfectionnements via la littérature scientifique (Pinheiro \& al., 2008) ou encore via des échanges directs avec des universitaires brésiliens ou encore américains avec qui ils collaborent depuis plusieurs années. En troisième lieu, nous avons vu que les laboratoires brésiliens ont graduellement modifié leurs spécifications, leurs procédés, leurs matières premières, dans le cours de la production. Le laboratoire fédéral a même développé une molécule dérivée de l'Efavirenz, qui a été testée à l'échelle du laboratoire de recherche et qui n'a pas été brevetée (entretien avec la responsable de la R\&D, Nubia Boechat, mai 2010).

Ce processus d'invention collective demeure toutefois limité aux deux dernières étapes de la synthèse du principe actif de l'Efavirenz. Les producteurs brésiliens sont encore dépendants des producteurs indiens à qui ils achètent un intermédiaire stratégique pour la synthèse de la molécule. Tandis que le laboratoire fédéral avait imaginé un programme de R\&D audacieux pour acquérir la synthèse complète de l'Efavirenz, un seul laboratoire du consortium s'est déclaré intéressé et a conduit des recherches séparées pour acquérir la technologie. Les laboratoires privés font état d'une échelle de production trop restreinte pour intégrer la production de cet intermédiaire complexe dans leurs murs et aussi de la facilité qu'il y a à l'importer dès lors que le produit acheté en Inde est de bonne qualité et disponible sans restrictions. Le laboratoire fédéral et le Ministère de la santé imaginent aujourd'hui de 
nouvelles coopérations avec le secteur pétrolier, avec l'entreprise publique Petrobras, pour surmonter ces problèmes d'échelle de production si une usine venait à produire des intermédiaires aussi bien pour la pharmacie que pour l'agrochimie.

\section{CONCLUSION}

L'état brésilien a joué un rôle essentiel pour autoriser et organiser la nationalisation de ce médicament, aussi bien par des décisions juridiques en matière de propriété intellectuelle, par les contrats de service par lesquels le laboratoire fédéral achète les principes actifs aux producteurs nationaux, ainsi que par les multiples actions du laboratoire fédéral pour coordonner le consortium et produire directement des technologies et le médicament final. Farmanguinhos a une double autorité dans ce processus : technique, pour élaborer des méthodes d'analyse et des spécifications qui seront distribuées à toutes les parties, étatique ou officielle lorsqu'il constitue le jury du consortium, sélectionne les producteurs de principe actif et négocie l'accord de consortium. Cet accord lui confie un rôle de surveillance sur les opérations de production des laboratoires privés. Par le moyen de ses rôles de producteur, de créateur de technologie, d'acteur de la propriété industrielle, de coordinateur de partenariats avec le secteur privé, de régulateur du prix du médicament générique et d'acteur dans le champ de la certification, le laboratoire fédéral, bras stratégique du Ministère de la Santé, concourt de manière essentielle à la formation de cette économie politique du médicament.

La trajectoire singulière de ce médicament participe à la relance d'une politique que l'on peut qualifier de "néo-développementiste » dès lors qu'il s'agit d'encourager l'acquisition des nouvelles technologies pharmaceutiques et de substituer des produits importés par des produits nationaux développés et fabriqués dans le cadre de Partenariats de Développement de Produits - PDP- qui associent laboratoires publics et laboratoires privés. La nouvelle loi de promotion des PDP de décembre 2010 autorise un surplus de prix temporaire pour l'acquisition par les laboratoires publics des matières premières pharmaceutiques brésiliennes dès lors que les firmes s'engagent à acquérir la technologie, à créer des emplois et à réduire leur prix dans un délai de 5 années. Cette politique néodéveloppementiste qui associe besoins domestiques de santé et acquisition de technologies avancées par les firmes brésiliennes pourrait illustrer la notion de «profitabilité sociale» proposée par Celso Furtado dans un article publié en 2000 dans la revue de la CEPAL. Dans l'esprit de Furtado la «profitabilité sociale» conjugue la politique nationale, le marché 
domestique, l'apprentissage technologique et la réduction des inégalités. L'Efavirenz brésilien réalise assez bien ce modèle de développement.

Bibliographie

Allen R, 1983, 1983, "Collective Invention", Journal of Economic Behavior and Organization, 4, 1-24 .

Arrow K, 1962, "The Economic Implications of Learning by Doing », The Review of Economic Studies, Vol. 29, Issue 3, 155-173.

Biehl J, 2009, « Accès au traitement du sida, marchés des médicaments et citoyenneté dans le Brésil d'aujourd'hui », Sciences Sociales et Santé, », Sciences Sociales et santé, n 3, vol 27, $13-46$.

Cassier M, 1998, "Le partage des connaissances dans les réseaux scientifiques : l'invention des règles de bonne conduite par les chercheurs" Revue française de Sociologie, 4, p 701-720.

Cassier M, Correa M, 2003, « Patents, Innovation and Public Health : Brazilian Public-Sector Laboratories'experience in copying AIDS drugs ", in Economics of AIDS and Access to HIV/AIDS Care in Developing Countries. Issues and Challenge, Ed. ANRS, p 89-107.

Cassier M, Correa M, 2009, «Eloge de la copie : le reverse engineering des antirétroviraux contre le VIH/sida dans les laboratoires pharmaceutiques brésiliens ", Sciences Sociales et Santé, n 3 , vol 27, 77-103.

Chaves G, Viera M, Reis R, 2008, « Access to medicines and intellectual property in Brazil : reflections and strategies of civil society », Sur.Revista Internacional de Direitos Humanos, vol.5, n8, 170-198.

Flynn M, 2007, «Brazil's Use of Compulsory Licenses for AIDS medicines », American Sociological Association, August 1-4, 1-20.

Furtado C, 1981, "Commentaires", in Technologie appropriée ou technologie sousdéveloppée”, E. Arghiri, PUF, 127-134.

Furtado C, 2000, Options for the future, Cepal Review, 70, 7-11.

Galvao J., 2002, Access to antiretroviral drugs in Brazil, The Lancet, volume 360, Issue 9348, 1862-1865.

Guennif S, 2012, «Brevet et Santé Publique dans les Pays du Sud : l'accès aux médicaments antisida », Etudes, vol. 416, n 7, 41-51.

Orsi F, Hasenclaver L, Fialho B, Tigre P, Coriat B, 2003, « Intellectual Property Rights, antiAids Policy and Generics Drugs. Lessons from the Brazilian Public Health Program », in Economics of AIDS and Access to HIV/AIDS Care in Developing Countries. Issues and Challenge, Ed. ANRS, 109-135. 
Pinheiro E, Antunes O, Fortunak J, 2008, «A survey of the syntheses of active pharmaceutical ingredients for antiretroviral drug combinations critical to access in emerging nations » Antiviral Research, 79, 143-165.

Possas C, 2008, "Compulsory Licensing in the real world: the case of ARV drugs in Brazil », in The Political Economy of HIV/AIDS in Developing Countries, Ed by B. Coriat, Edward Elgar, London, 150-166.

Rosenberg S, 2010, Using the Trade-related Aspects of Intellectual Property Rights Agreement to Promote Public Health : A comparative analysis of compulsory licensing in Thailand and Brazil, Master thesis, EHESS, 129 pages.

Teixeira P, Vitoria MC, Barcarolo J, 2003, The Brazilian experience in providing universal access to antiretroviral therapy, In :Moatti J.-P., Coriat B., Souteyrand Y., Barnett T., Dumoulin J., Flori Y.-A., eds, Economics of AIDS and access to HIV/AIDS, 69-88.

Velasquez G, Correa C, Thirukumaran Balasubramaniam, 2004, «WHO in the Frontlines of the Access to Medicines Battle: The Debate on Intellectual Property Rights and Public Health ", Intellectual Property in the context of the WTO TRIPS Agreement ", Eds J. Bermudez, MA Oliviera, Fiocruz, 83- 97. 